\title{
Visual field meridians modulate the reallocation of object-based attention
}

\author{
Adam J. Barnas ${ }^{1}$ • Adam S. Greenberg ${ }^{1}$
}

Published online: 5 May 2016

(C) The Psychonomic Society, Inc. 2016

\begin{abstract}
Object-based attention (OBA) enhances processing within the boundaries of a selected object. Larger OBA effects have been observed for horizontal compared to vertical rectangles, which were eliminated when controlling for attention shifts across the visual field meridians. We aimed to elucidate the modulatory role of the meridians on OBA. We hypothesized that the contralateral organization of visual cortex accounts for these differences in OBA prioritization. Participants viewed "L"-shaped objects and, following a peripheral cue at the object vertex, detected the presence of a target at the cued location (valid), or at a non-cued location (invalid) offset either horizontally or vertically. In Experiment 1 , the single displayed object contained components crossing both meridians. In Experiment 2, one cued object and one non-cued object were displayed such that both crossed the meridians. In Experiment 3, one cued object was sequestered into one screen quadrant, with its vertex either near or far from fixation. Results from Experiments 1 and 2 revealed a horizontal shift advantage (faster RTs for horizontal shifts across the vertical meridian compared to vertical shifts across the horizontal meridian), regardless of whether shifts take place within a cued object (Experiment 1) or between objects (Experiment 2). Results from Experiment 3 revealed no difference between horizontal and vertical shifts for objects that were positioned far from fixation, although the horizontal shift advantage reappeared for objects near fixation. These findings suggest a critical modulatory role of visual field meridians in the efficiency of reorienting object-based attention.
\end{abstract}

Adam S. Greenberg

agreenb@uwm.edu

1 Department of Psychology, University of Wisconsin-Milwaukee, Milwaukee, WI 53201, USA
Keywords Object-based attention $\cdot$ Horizontal-vertical anisotropy $\cdot$ Visual field meridians $\cdot$ Object selection

\section{Introduction}

Because of the known neurophysiological spatial receptive field organization of the visual system, attention is thought to be primarily space-based; that is, the information to which one attends is selected based on its location in the visual field. As a result, attention directed to these spatial locations allows an individual to more deeply and efficiently process selected information (Posner, 1980; Posner, Snyder, \& Davidson, 1980). However, it has been demonstrated that the representational basis of attentional selection can also be object-based (Duncan, 1984; Egly, Driver, \& Rafal, 1994). Object-based attention (OBA) generally leads to a preferential and simultaneous processing of visual information that is typically characterized by faster RTs and heightened accuracy to targets contained within the boundaries of an attended object compared to targets contained within the boundaries of an unattended object, otherwise known as a same-object advantage. As a result, information processing within the boundaries of an attended object occurs more rapidly compared with the information processing that occurs between objects.

In the first demonstration of object-based attention, observers were instructed to report pairs of features that exist on a single object or on two different objects. Observers were more accurate and faster at reporting pairs of features of the same object as opposed to pairs of features spanning different objects, demonstrating an attentional cost incurred from shifting attention away from the attended object (Duncan, 1984). A task was later developed in which both spacebased and object-based attention could be measured simultaneously using a double-rectangle cueing paradigm (Egly, 
Driver, and Rafal, 1994). Shifts of attention within two parallel rectangles (oriented either vertically, to the left and right of the vertical meridian; or horizontally, above and below the horizontal meridian) are contrasted against shifts of attention between rectangles. In this task, a spatial cue (typically, a brightening of one end of one rectangle) appears briefly, after which a single target appears in one of three possible locations on the objects: (1) the cued location of the cued rectangle ("Valid location"), (2) a non-cued location of the cued rectangle (Invalid-same location), or (3) a non-cued location of the non-cued rectangle (Invalid-different location). Critically, the two invalid target locations are equidistant from the cue and, thus, allow for the measurement of object-based attention. Observers are faster to detect targets at the valid location on the cued rectangle compared with targets at the invalid-same location, a demonstration of space-based attentional selection. Critically, observers are also faster to detect targets at the invalid-same location than the invalid-different location, indicating that the cue draws observers' attention to aspects of the cued object (not simply the cued location), producing an object-based attention effect that cannot be explained solely by space-based attention, since the invalid targets are equidistant from the cue.

Recent efforts have moved away from simply documenting instances of object-based attention to understanding the mechanisms that underlie object-based attentional selection. One postulated theory is the sensory enhancement account. Under this view, attention (in the form of a spatial gradient) is first centered on a cued location, which is followed by an automatic spreading of attentional resources within the boundaries of the cued object, ultimately improving the quality of an early sensory representation of the cued object as a whole (Chen \& Cave, 2006, 2008; Richard, Lee, \& Vecera, 2008). As a result, visual information within the boundaries of the cued object is enhanced relative to visual information within the boundaries of unattended objects due to biased competition (Desimone \& Duncan, 1995). A second theory that was proposed to underlie object-based attentional selection is the object-specific attentional prioritization account (Shomstein \& Yantis, 2002, 2004), in which target locations within an attended object are afforded higher priority than target locations in unattended objects. This is accomplished via an automatic spatial selection of a cued location and subsequent prioritization of attention from the cued location to areas in which the probability of the target appearing is higher (i.e., in the cued object) over locations in which the probability of the target appearing is lower (i.e., in the non-cued object). Under this view, object-based attention effects result from the unequal prioritization of attention to the invalid-same and invalid-different locations. As such, this explanation ultimately establishes the order in which an observer will search a visual display for the presence of a target, beginning first at the valid location, next at the invalid-same location, and finally at the invalid-different location (Greenberg et al., 2015). However, the attentional prioritization strategy is not as rudimentary as a visual search mechanism, because although both processes rely on the combined attentional priorities of items (Shomstein \& Yantis, 2002; Wolfe, 1994), the former approach is further constrained by the perceptual objectness formed by the object's boundaries (Shomstein, 2012; Greenberg et al., 2015).

A recent study (Pilz, Roggeveen, Creighton, Bennett, \& Sekuler, 2012) demonstrated that the preferential processing of visual information as a result of object-based attention was modulated by the orientation of the two parallel rectangles. In this experiment, a large number of observers were presented with the double-rectangle cueing paradigm (Egly, Driver, \& Rafal, 1994) and performed either a detection task or a discrimination task. Space-based attention effects were observed in horizontal and vertical rectangles, as evidenced by increased accuracy and faster RTs to the valid location compared with the invalid-same location. Object-based attention effects, however, were relatively small compared with the space-based effects and varied as a function of rectangle orientation. Overall RTs were also significantly slower for horizontally oriented rectangles compared with vertically oriented rectangles. Moreover, object-based attention effects were not observed (at the group level) for vertically oriented rectangles across three different experiments. This differs from many previous reports of object-based attention effects (for a review, see Chen, 2012) that do not show an effect of, or (generally) even explicitly test for, object orientation. The results of Pilz and colleagues (2012) showed that, for horizontally oriented rectangles, RTs to the invalid-same location were significantly faster than RTs to the invalid-different location, the same-object advantage that typically characterizes object-based attention effects. However, they also showed that, for vertically oriented rectangles, RTs to the invalid-same location were actually slower than RTs to the invalid-different location. This same-object cost in vertically oriented rectangles has occasionally been documented by others, as well (Davis \& Holmes, 2005; Harrison \& Feldman, 2009; Chen \& Huang, 2015). To explain this effect of rectangle orientation on the same-object advantage, Pilz and colleagues (2012) postulated that attention may be more efficiently allocated parallel to the horizontal meridian than the vertical meridian, a phenomenon previously observed in visual search studies (Carrasco, Evert, Chang, \& Katz, 1995; MacKeben, 1999). Recent work from our laboratory showed that the orientation effects observed in the Pilz et al. (2012) study disappear when controlling for shifts across the visual field meridians (Greenberg, et al., 2014). This suggests that effects of the meridians may be the cause of orientation 
differences reported in object-based attention studies using the double-rectangle cueing paradigm.

Anatomical and physiological evidence, however, may also provide an explanation for differences between the horizontal and vertical dimensions. It is well established that the left and right visual field representations are organized contralaterally. As a result, objects that appear in both visual hemifields, crossing the vertical meridian, are partially represented in corresponding retinotopic areas in the left and right cortical hemispheres; whereas objects appearing entirely within a single visual hemifield are represented fully in the corresponding contralateral hemisphere. Consequently, attention allocation along the horizontal meridian may be impaired due to an interhemispheric boundary imposed by the contralateral organization of visual space in the cortical hemispheres. Shifting attention horizontally across the vertical screen meridian may require hemispheric interactions and additional cortical processing that is not required when attention shifts occur entirely within a hemifield (Holtzman, Sidtis, Volpe, Wilson, \& Gazzaniga, 1981; Reuter-Lorenz \& Fendrich, 1992a).

However, lower and upper visual field representations of retinotopic areas within extrastriate cortex are also sequestered physiologically. Representations of the lower half of the visual field correspond to dorsal aspects of retinotopic visual cortex, whereas representations of the upper visual field correspond to ventral aspects of retinotopic visual cortex (Van Essen, 1985). Therefore, the horizontal meridian represents an intrahemispheric boundary (Sereno \& Kosslyn, 1991), which may require additional cortical processing when shifting attention between upper and lower visual field representations. Our goal was to examine, behaviorally, whether either (or both) of these physiological segregations of the visual field can explain the observed effects of object orientation on OBA. To accomplish this, we measured shifts of attention from cued locations to invalid locations that either crossed or did not cross the vertical and horizontal screen meridians and how these shifts of attention varied within a single object and between two objects.

\section{Experiment 1}

The purpose of Experiment 1 was to investigate the manner by which object-based attention within an object is reallocated across the vertical and horizontal meridians of the visual field. We modified the standard double-rectangle cuing paradigm developed by Egly and colleagues (1994) to investigate this aim. Rather than utilizing the original paradigm in which shifts within a cued rectangle are contrasted against shifts between a cued and non-cued rectangle, we opted for an object that permitted both vertical and horizontal shifts of attention across the screen meridians to be contained within the boundaries of a single object. Importantly, this feature avoids confounding shift direction across the meridians with object selection, as only a single object was ever available for selection. Of interest to this experiment were the differences between response latencies to targets that were presented in invalidly cued locations along the horizontal and vertical meridian of the same object. As outlined earlier, based on interhemispheric boundaries within visual cortex, one would hypothesize that response times (RTs) to invalidly cued locations in the vertical dimension will be faster than RTs to invalidly cued locations in the horizontal dimension. However, based on intrahemispheric boundaries within visual cortex, one would predict that vertical RTs would be slower than horizontal RTs.

\section{Method}

\section{Participants}

Forty-six participants from the University of WisconsinMilwaukee (UWM) and surrounding community $\left(M_{\text {age }}=\right.$ 24.22 years, $S D_{\text {age }}=8.65$ years; 31 women, 15 men) took part in this experiment, which was approved by the UWM Institutional Review Board. All participants provided written, informed consent before the start of the experiment and indicated that they had normal or corrected-to-normal visual acuity. As compensation for their participation, volunteers had the option of receiving 1 hour of extra credit toward a psychology course or the standard hourly pay rate.

\section{Apparatus and stimuli}

All stimuli were presented using a 17-in, CRT monitor $(1,024$ $\times 768$ pixel screen resolution, $100-\mathrm{Hz}$ refresh rate) driven by an Apple Mac Mini computer running OS X (Version 10.8.5). Stimuli were programmed in the GNU Octave software platform (Bateman et al., 2015) using Psychophysics Toolbox extensions (Brainard, 1997; Pelli, 1997). Participants performed the experiment in a dimly lit room. A chin rest was used to support and stabilize participants' heads at a distance of approximately $58 \mathrm{~cm}$.

As shown in Fig. 1, subjects viewed a single gray object (RGB: [128 128 128]) that consisted of a vertical rectangle $\left(2.0^{\circ} \times 12.0^{\circ}\right)$ joined with a horizontal rectangle $\left(12.0^{\circ} \times\right.$ $2.0^{\circ}$ ), forming a unified L-shaped object, on a black background. While participants fixated centrally on a white fixation cross $\left(0.2^{\circ} \times 0.2^{\circ}\right)$ of a fixed-width font (Monaco, font size 20 ), the object vertex was randomly positioned in one screen quadrant such that one object component always crossed the vertical meridian and the other component always crossed the horizontal meridian. The L-shaped object was centered on the screen and around the central fixation cross, such that the distances between the vertical screen meridian to 
Fig. 1 Trial sequence in Experiment 1 for a cued gray Lshaped object in the upper-left quadrant. (A-C) Three possible trial conditions defined by the location of the blue target " $\mathrm{T}$ " in relation to the red peripheral cue

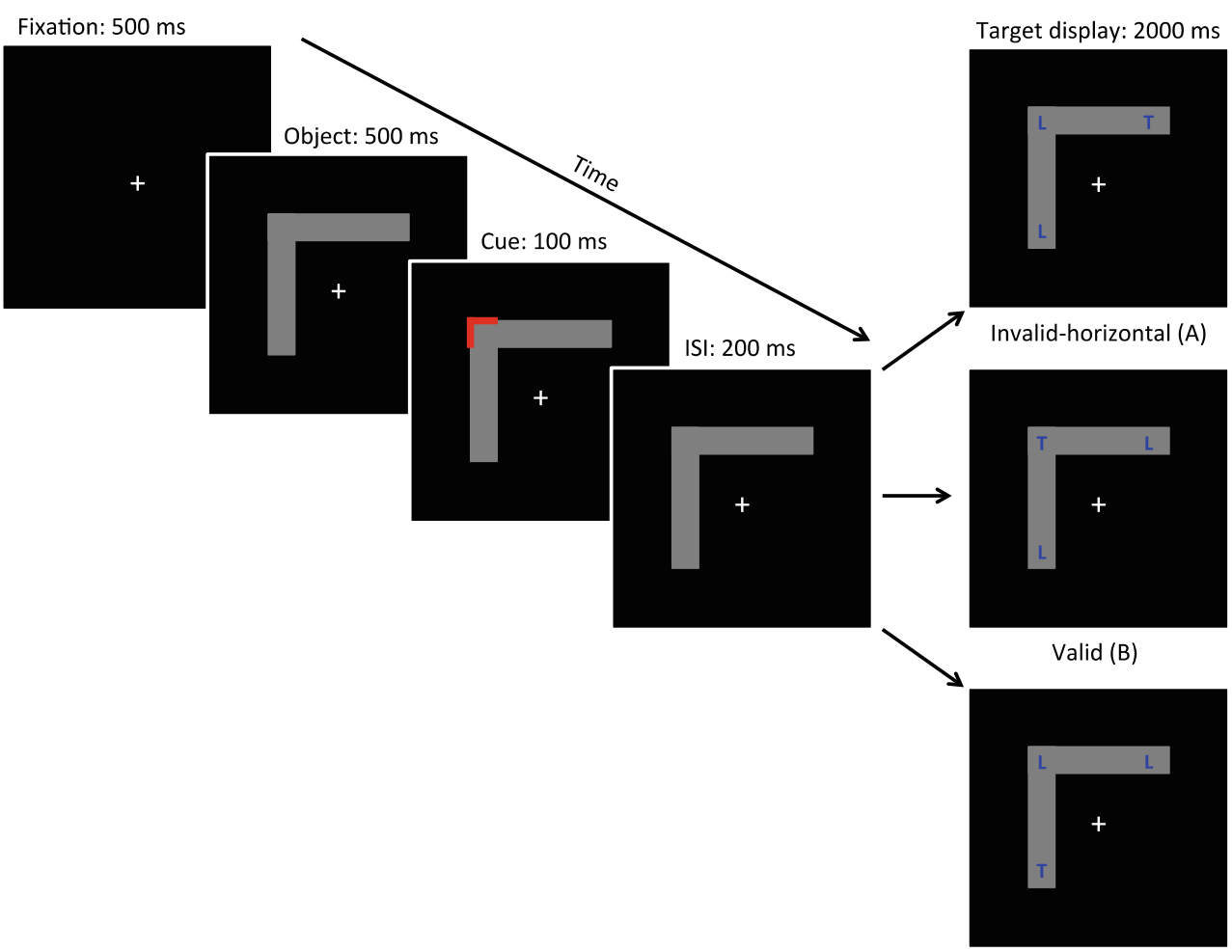

Invalid-vertical (C) the inner edge of the vertical component rectangle, and between the horizontal screen meridian to the inner edge of the horizontal component rectangle, were both $4.0^{\circ}$.

The red exogenous cue (RGB: [255 00 0]) also consisted of a vertical rectangle $\left(0.34^{\circ} \times 2.0^{\circ}\right)$ joined with a horizontal rectangle $\left(2.0^{\circ} \times 0.34^{\circ}\right)$ and always appeared around the outer edge of the object vertex. The target display consisted of blue letters (RGB: [0 0 255]; Monaco, font size 20) subtending $0.67^{\circ}$ in length and width and consisted of a single target (the letter T) among two non-targets (the letter L). Target letters were centered left-to-right within the vertical object component and top-to-bottom within the horizontal object component; targets were positioned so that their centers were $1.0^{\circ}$ from the near end of the object.

\section{Design}

The following three trial types were defined by the location of the target "T" at: (1) the cued location of the object vertex (valid condition), (2) the non-cued location of the object's horizontal component (invalid-horizontal condition), or (3) the non-cued location of the object's vertical component (invalid-vertical condition). Targets on invalid-horizontal trials and invalid-vertical trials were equidistant from the cue. There were 8 blocks of trials, each containing 120 trials for a total of 960 trials. Each block consisted of $60 \%$ valid trials ( 72 trials per block; 576 total), 10\% invalid-horizontal trials (12 trials per block; 96 total), and 10\% invalid-vertical trials (12 trials per block; 96 total). The remaining condition was composed of "catch trials" (20\%; 24 trials per block; 192 total), in which only non-target letters appeared on the object.

\section{Procedure}

Before starting the experiment, participants were instructed to maintain fixation on the white fixation cross throughout the duration of the trial. As shown in Fig. 1, participants were first presented with a fixation cross for $500 \mathrm{~ms}$. The object was then added to the display for $500 \mathrm{~ms}$, which was immediately followed by the addition of the red cue. The cue was presented for $100 \mathrm{~ms}$, and after a 200-ms interstimulus interval from the offset of the cue, the target display appeared. The target " $T$ " randomly appeared in one of the three possible locations (excluding catch trials). Nontargets also appeared on the object in all locations unoccupied by the target. Participants performed a detection task in which they responded to the presence of the target letter, and RTs were recorded. Participants were instructed to respond as quickly as possible and to withhold their response during catch trials.

\section{Results and discussion}

Before conducting any statistical analyses, participants with false alarms (responding in the absence of a target) to more than 19 catch trials (or 10\%) were removed from the original 
sample of 46 participants, because a false-alarm rate of this magnitude indicates that a participant is not successfully engaged in the task. This resulted in a final sample of 32 participants $\left(M_{\text {age }}=25.00\right.$ years, $S D_{\text {age }}=10.15$ years; 23 women, 9 men) with a mean false-alarm rate of 10 catch trials $(S D=6)$. Anticipatory responses $(<200 \mathrm{~ms})$ and misses (failing to respond to the presence of the target) were excluded from the individual subject data. Participants, on average, failed to respond to $3.16 \%$ of valid trials $(S E M=0.61 \%), 3.75 \%$ of invalid-horizontal trials $(S E M=0.84 \%)$, and $4.31 \%$ of invalid-vertical trials $(S E M=0.69 \%)$.

Mean RT differences were calculated by subtracting mean raw RTs to targets in the valid location $(M=562.93 \mathrm{~ms})$ from mean raw RTs to targets in each invalid location to quantify participants' reallocation of attention within an object across the vertical and horizontal meridians. These mean RT differences were then subjected to a within-subjects, repeated measures ANOVA with Shift Direction (vertical, horizontal) as a single factor. Results revealed a significant effect of Shift Direction, $F(1,31)=79.87, p \leq 0.001, \eta_{\mathrm{p}}{ }^{2}=0.72$, indicating a difference in invalid target detection when reallocating attention horizontally $(M=162.35 \mathrm{~ms})$ versus vertically $(M=$ $240.75 \mathrm{~ms}$; Table 1). A similar main effect of Shift Direction $(p \leq 0.001)$ was found when all 46 participants were analyzed together.

The results from Experiment 1 revealed that shifts of object-based attention contained within the boundaries of a single cued L-shaped object (with one component crossing the vertical meridian and the other component crossing the horizontal meridian) were allocated more efficiently along the horizontal meridian than along the vertical meridian. This result supports the hypothesis that the additional cortical processing resulting from the intrahemispheric segregation of the upper and lower visual hemifields impairs the efficiency of attention reallocation across the horizontal meridian versus the vertical meridian. Thus, intrahemispheric cortical representations of visual information are seemingly more costly than interhemispheric representations of visual information. These results support the conclusions of Pilz and colleagues (2012). However, because only a single object was presented in Experiment 1, there was no competition for attentional selection, making it possible that few object-based attentional resources were necessary to perform the task. Therefore, Experiment 2 was designed to observe whether similar costs resulting from inter- and intrahemispheric boundaries occurred under competitive conditions of object-based selection.

\section{Experiment 2}

The purpose of Experiment 2 was to investigate the manner in which object-based attention is reallocated across the vertical meridian and the horizontal meridian in the presence of two perceptual objects. In this experiment, we used two objects, as is typical in investigations of OBA (Chen, 2012), introducing competition for attentional resources between objects. By using two objects, we were now able to look at differences in vertical and horizontal orienting for not only the cued object but also for a non-cued object. We predicted, based on the results of Experiment 1, that reallocating attention across the vertical meridian would be more efficient than reallocating attention across the horizontal meridian, regardless of which object was selected. To accomplish this aim, we displayed two L-shaped objects simultaneously, the vertices of which were always positioned in diagonally opposing screen quadrants.

\section{Method}

\section{Participants}

Thirty-four participants from UWM and the surrounding community $\left(M_{\text {age }}=24.30\right.$ years, $S D_{\text {age }}=6.45$ years [one participant did not provide age]; 26 women, 8 men) took part in this experiment, which was approved by the UWM Institutional Review Board. All participants provided written, informed consent before the start of the experiment and indicated that they had normal or corrected-to-normal vision. As compensation for their participation, volunteers had the option of receiving 1 hour of extra credit toward a psychology course or the standard hourly pay rate.

\section{Apparatus and stimuli}

All aspects of Experiment 2 were identical to those of Experiment 1, with the following two exceptions (Fig. 2). First, participants were presented with two L-shaped objects. One object maintained the same physical dimensions as the object in Experiment 1, whereas the component rectangles of the second object were 3 degrees longer $\left(2.0^{\circ} \times 15.0^{\circ}\right)$. The inner edges of the two objects were separated by $1.0^{\circ}$. While participants fixated centrally, the two object vertices were positioned in diagonally opposing quadrants around the central fixation cross. The two L-shaped objects were centered on the screen and around the fixation cross, such that the distances between the vertical screen meridian to the inner edge of the vertical component rectangle of either object and between the horizontal screen meridian to the inner edge of the horizontal component rectangle of either object were both $5.5^{\circ}$.

Second, the target display consisted of a single target (the letter $\mathrm{T}$ ) among four nontargets (the letter L) that were centered vertically and horizontally within each object at five locations. One letter of the target display always appeared in the vertex of the cued object. The remaining four letters were centered left-to-right within the vertical object component and top-to-bottom within the horizontal object component in both 
Table 1 Mean response time differences (msec) for Experiments 1-3

\begin{tabular}{lllllll}
\hline & Exp 1 & Exp 2 & & & Exp 3 & \\
\cline { 2 - 4 } \cline { 6 - 7 } & Cued & Cued & Non-cued & & Near-cued & Far-cued \\
\hline Invalid-vertical & $240.75(4.39)$ & $299.38(11.78)$ & $448.02(10.05)$ & & $297.52(5.67)$ & $183.38(7.41)$ \\
Invalid-horizontal & $162.35(4.39)$ & $214.89(10.99)$ & $328.02(9.38)$ & & $235.46(5.67)$ & $163.82(7.41)$ \\
Difference & $78.40 *(8.77)$ & $84.49 *(20.03)$ & $120.00 *(18.47)$ & & $62.06 *(11.34)$ & $19.56(14.81)$ \\
\hline
\end{tabular}

RT differences were calculated by subtracting mean RTs to valid targets from mean RTs to targets in each invalid location. Values in parentheses are SEMs. Asterisks indicate horizontal-vertical anisotropy (significant difference from zero; all $p \mathrm{~s} \leq 0.001$ ) the cued and non-cued objects; targets were positioned so that their centers were $1.0^{\circ}$ from the near end of the object.

\section{Design}

The following five trial types were defined by the location of the target "T" at: (1) the cued location of the object vertex (valid condition), (2) the non-cued location of the cued object's horizontal component (invalid-horizontal cued condition), (3) the non-cued location of the cued object's vertical component (invalid-vertical cued condition), (4) the non-cued location of the non-cued object's horizontal component (invalid-horizontal non-cued condition), and (5) the non-cued location of the non-cued object's vertical component (invalid-vertical non-cued condition). Targets in invalid-horizontal locations and invalid-vertical locations for both cued and noncued objects were equidistant from the cue. There were 6 blocks of trials, each containing 160 trials for a total of 960 trials. Each block consisted of $60 \%$ valid trials (96 trials per block; 576 total), $5 \%$ invalid-horizontal cued trials ( 8 trials per block; 48 total), 5\% invalid-vertical cued trials ( 8 trials per block; 48 total), 5\% invalid-horizontal non-cued trials ( 8 trials per block; 48 total), and 5\% invalid-vertical non-cued trials ( 8 trials per block; 48 total). The remaining condition was composed of "catch trials" (20\%; 32 trials per block; 192 total).

\section{Procedure}

The procedure was identical to that of Experiment 1, with the only exception that the target " $\mathrm{T}$ " now randomly appeared in one of five possible locations (except on catch trials) on the cued object (3 locations) or the non-cued object (2 locations). Non-targets also appeared on the objects in the locations unoccupied by the target. Participants performed a detection task in which they responded to the presence of the target letter, and RTs were recorded. Participants were instructed to
Fig. 2 Trial sequence in Experiment 2 for a cued gray Lshaped object in the upper-left quadrant and a non-cued gray Lshaped object in the lower-right quadrant. (A-E) Five possible trial conditions defined by the location of the blue target " $T$ " in relation to the red peripheral cue

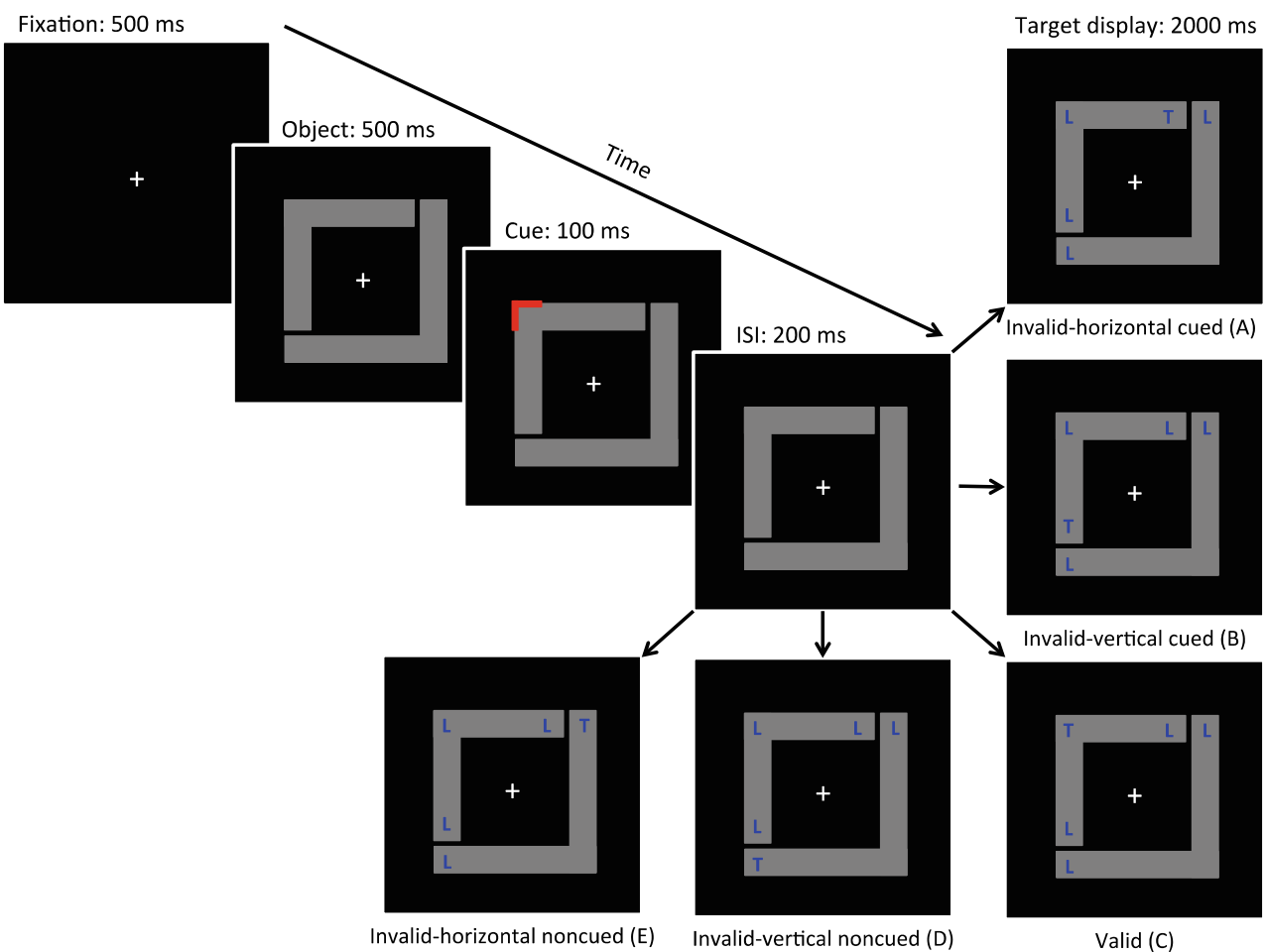


respond as quickly as possible and to withhold their response during catch trials.

\section{Results and discussion}

As in Experiment 1, participants with false alarms to more than 19 catch trials were removed from the original sample of 34 participants before conducting any statistical analyses. This resulted in a final sample of 30 participants $\left(M_{\text {age }}=24.55\right.$ years, $S D_{\text {age }}=6.75$ years; 22 women, 8 men) with a mean false-alarm rate of 13 catch trials $(S D=5)$. Anticipatory responses $(<200 \mathrm{~ms})$ and misses (failing to respond to the presence of the target) were excluded from the individual subject data. Participants, on average, failed to respond to $2.24 \%$ of valid trials $(S E M=0.40 \%), 5.04 \%$ of invalid-horizontal cued trials $(S E M=0.80 \%), 5.27 \%$ of invalid-vertical cued trials $(S E M=1.07 \%), 4.83 \%$ of invalid-horizontal non-cued trials $(S E M=1.00 \%)$, and $10.67 \%$ of invalid-vertical non-cued trials $(S E M=3.46 \%)$.

Mean RT differences were calculated by subtracting mean raw RTs to valid targets $(M=564.26 \mathrm{~ms})$ from mean raw RTs to targets in each invalid location and submitted to a $2 \times 2$ repeated measures ANOVA with Object Validity (cued, noncued) and Shift Direction (vertical, horizontal) as withinsubjects factors. The analysis revealed a main effect of Object Validity, $F(1,29)=171.32, p \leq 0.001, \eta_{\mathrm{p}}{ }^{2}=0.86$, indicating a significant difference in invalid target detection when object-based attention was reallocated to invalid targets within the boundary of the cued object $(M=257.13 \mathrm{~ms})$ versus the non-cued object $(M=388.02 \mathrm{~ms})$. The analysis also revealed a significant main effect of Shift Direction, $F(1,29)=$ 29.20, $p \leq 0.001, \eta_{\mathrm{p}}{ }^{2}=0.50$, which indicated a significant difference in invalid target detection when reallocating attention horizontally $(M=271.46 \mathrm{~ms})$ versus vertically $(M=$ $373.70 \mathrm{~ms})$. These main effects were further qualified by a significant two-way interaction, $F(1,29)=5.70, p=0.024$, $\eta_{\mathrm{p}}{ }^{2}=0.16$. Similar main effects of Object Validity and Shift Direction $(p s \leq 0.001)$, as well as the interaction $(p=0.004)$ were found when all 34 participants were analyzed together.

The interaction between Object Validity and Shift Direction describes the significant differences in the shift direction magnitude as a function of object type (Fig. 3). Paired samples $t$ tests revealed a larger shift direction effect when the invalid targets appeared along the horizontal meridian of the cued object $(M=214.89 \mathrm{~ms})$ versus the vertical meridian ( $M=$ $299.38 \mathrm{~ms}$ ), $t(29)=3.83, p=0.001$. We also observed (in the non-cued object) a larger shift direction effect for invalid targets along the horizontal meridian $(M=328.02 \mathrm{~ms})$ versus the vertical meridian $(M=448.02 \mathrm{~ms}), t(29)=6.50, p \leq 0.001$. Importantly, as shown in Table 1, this interaction is driven by a larger anisotropy between horizontal and vertical shifts for the

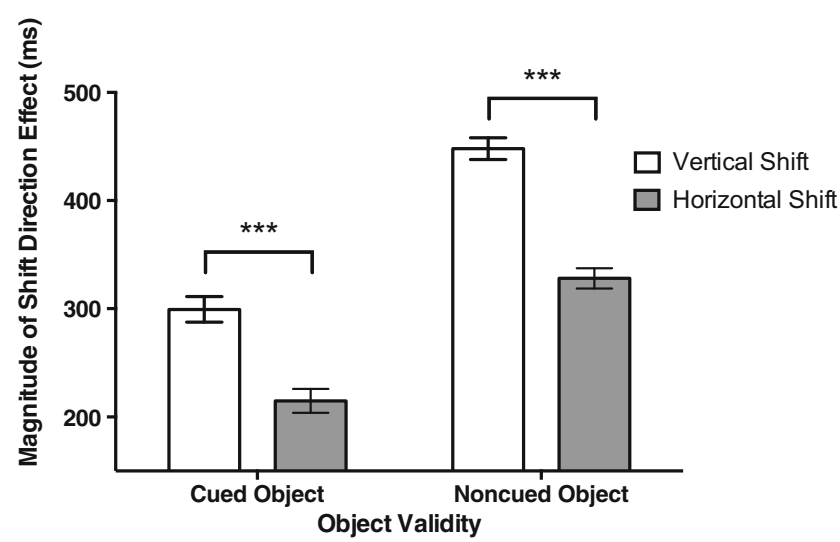

Fig. 3 Mean response time data ("Magnitude of Shift Direction Effect") measured for the Object Validity x Shift Direction interaction in Experiment 2

non-cued object (120.00 ms) versus the cued object (84.49 $\mathrm{ms})$.

One might question whether this paradigm used in Experiment 2 measured object-based attention in the "traditional" sense (i.e., a same-object advantage). We have purposely chosen to stray from the traditional OBA paradigm because our goal was to measure the asymmetry between horizontal and vertical shifts of attention without the confound of shifting between objects. In the standard double-rectangle cueing paradigm (Egly, Driver, \& Rafal, 1994) with parallel vertical rectangles, for example, a vertical shift of attention is restricted to the boundaries of the cued object, whereas a horizontal shift of attention must cross from the cued object to the non-cued object. The same is true for horizontal rectangles; however, in this scenario, a vertical shift of attention must cross object boundaries, whereas a horizontal shift does not cross object boundaries. In Experiment 2, all components of a traditional object-based attention paradigm were present (i.e., cue, valid target location on a cued object, invalid target locations on a cued object, invalid target locations on a noncued object, within-object shifts of attention, and betweenobject shifts of attention). Our paradigm differs from the double-rectangle cuing paradigm in that we utilized two Lshaped objects rather than the two parallel rectangles. Moreover, our main interest lies in the asymmetry between horizontal and vertical shifts of attention rather than the same-object advantage metric typically employed. Nevertheless, Experiment 2 does provide a means by which to measure a form of same-object advantage. We began by calculating the average shift velocity (time/distance) from valid to invalid-horizontal locations on the cued object. We then used this velocity measure to predict the expected mean RT to the invalid-horizontal locations on the non-cued object $\left(M_{\text {predicted }}=268.61 \mathrm{~ms}\right)$. This allowed us to compare whether shift velocity was affected by shifting away from the cued object. That is, if the predicted invalid non-cued object RT matched the measured RT, then we can conclude that 
participants were unaffected by the shift to a new object, because there was no change in the velocity of the shift. Whereas if the predicted RT was significantly different from the measured RT, we can conclude that participants were affected by the shift to a new object, and thus, attention must have selected the cued object in an object-based manner. The results of a one-sample $t$ test showed that measured horizontal shifts to non-cued objects $\left(M_{\text {measured }}=328.02 \mathrm{~ms}\right)$ were slower than predicted by the cued object velocity, $t(29)=3.21, p=0.003$. This advantage was also present for vertical shifts: measured vertical shifts to non-cued objects $\left(M_{\text {measured }}=448.02 \mathrm{~ms}\right)$ were slower than predicted vertical shifts to non-cued objects $\left(M_{\text {predicted }}=374.23 \mathrm{~ms}\right), t(29)=2.68, p=0.012$. These analyses suggest that there is an advantage for shifting within a cued object. Therefore, we conclude that this paradigm does, in fact, engage a mechanism of object-based selection akin to that of traditional OBA paradigms.

Additionally, as in Experiment 1, horizontal shifts (across the vertical meridian) were significantly faster than vertical shifts (across the horizontal meridian), here observed for both the cued object and non-cued object. The anisotropy between reallocating attention to invalid-horizontal targets and invalidvertical targets, however, was larger for the non-cued object than the cued object despite the fact that the invalid locations were equidistant (within a given object) from the cue. That is, the difference between horizontal and vertical shifts to invalid locations for both the cued and non-cued objects should, in theory, be equivalent. The larger anisotropy for the non-cued object suggests that the effect of meridian crossings is enhanced with respect to shifts of attention away from the cued object. ${ }^{1}$ Finally, mirroring the results of Experiment 1, a cost was incurred in the vertical dimension (relative to horizontal), which we attribute to the intrahemispheric boundary within retinotopic visual cortex.

\section{Experiment 3}

In the third experiment, we measured the reallocation of attention with a single cued object in which shifts of attention in either direction are not confounded with shifts across the vertical or horizontal meridians. To accomplish this, a single Lshaped object was presented entirely within one screen

\footnotetext{
${ }^{1}$ This conclusion was drawn by comparing the horizontal-vertical anisotropies for the cued $(M=84.49 \mathrm{~ms})$ and non-cued object $(M=120.00 \mathrm{~ms})$, $t(29)=2.39, p=0.024$. Alternatively, it is possible that this effect was driven partly by longer RTs in the non-cued object condition compared with the cued object condition (e.g., the invalid-horizontal shift of attention was $214.89 \mathrm{~ms}$ in the cued object condition and $328.02 \mathrm{~ms}$ in the non-cued object condition). In terms of proportionate costs for the invalid-vertical shift relative to the invalid-horizontal shift, the invalidvertical shift of attention ( $M=299.38 \mathrm{~ms})$ cost $39 \%$ more than the invalidhorizontal shift in the cued object condition and 37\% more in the noncued object condition $(M=448.02 \mathrm{~ms})$.
}

quadrant. Sequestering the object into a single quadrant allows the horizontal dimension of the object to be represented entirely by the contralateral hemisphere; thus, there should be no additional processing required as a result of crossing the interhemispheric boundary. Furthermore, because the vertical dimension of the object was sequestered into either the upper or lower visual field, there should be no additional processing required as a result of crossing the intrahemispheric boundary. We predicted that the lack of any meridian crossings should lead to no anisotropy between reorienting object-based attention horizontally and vertically within an object.

\section{Method}

\section{Participants}

Forty-one participants from UWM and the surrounding community $\left(M_{\text {age }}=23.39\right.$ years, $S D_{\text {age }}=6.81$ years; 27 women, 14 men) took part in this experiment, which was approved by the UWM Institutional Review Board. All participants provided written, informed consent before the start of the experiment and indicated that they had normal or corrected-to-normal vision. As compensation for their participation, all volunteers had the option of receiving 1 hour of extra credit toward a psychology course or the standard hourly pay rate.

\section{Apparatus, stimuli, design, and procedure}

All aspects of Experiment 3 were identical to those of Experiment 1, with the following three exceptions (Fig. 4). First, the component rectangles of the L-shaped object had dimensions of $2.0^{\circ} \times 10.65^{\circ}$. The object was positioned such that its nearest edge was $0.66^{\circ}$ above or below the horizontal meridian and $0.66^{\circ}$ to the left or to the right of the vertical meridian.

Second, the object vertex was positioned within each screen quadrant such that it was either near fixation or far from fixation, randomly. The near-cued L-shaped object was positioned on the screen such that the distances between the vertical screen meridian to the inner edge of the vertical component rectangle and between the horizontal screen meridian to the inner edge of the horizontal component rectangle were both $0.66^{\circ}$. The vertex of the near-cued L-shaped object was $0.93^{\circ}$ away from the central fixation cross. The far-cued Lshaped object was positioned on the screen such that the distances between the vertical screen meridian to the inner edge of the vertical component rectangle and between the horizontal screen meridian to the inner edge of the horizontal component rectangle were both $9.3^{\circ}$.

Third, there were 6 blocks of trials, each containing 160 trials for a total of 960 trials. Each block consisted of $60 \%$ valid trials (96 trials per block; 576 total), 10\% invalid- 
Fig. 4 Trial sequence in Experiment 3 for a far cued gray L-shaped object (a) and a near cued gray L-shaped object (b) in the upper-left quadrant. (A-C)

Three possible trial conditions defined by the location of the blue target " $T$ " in relation to the red peripheral cue a

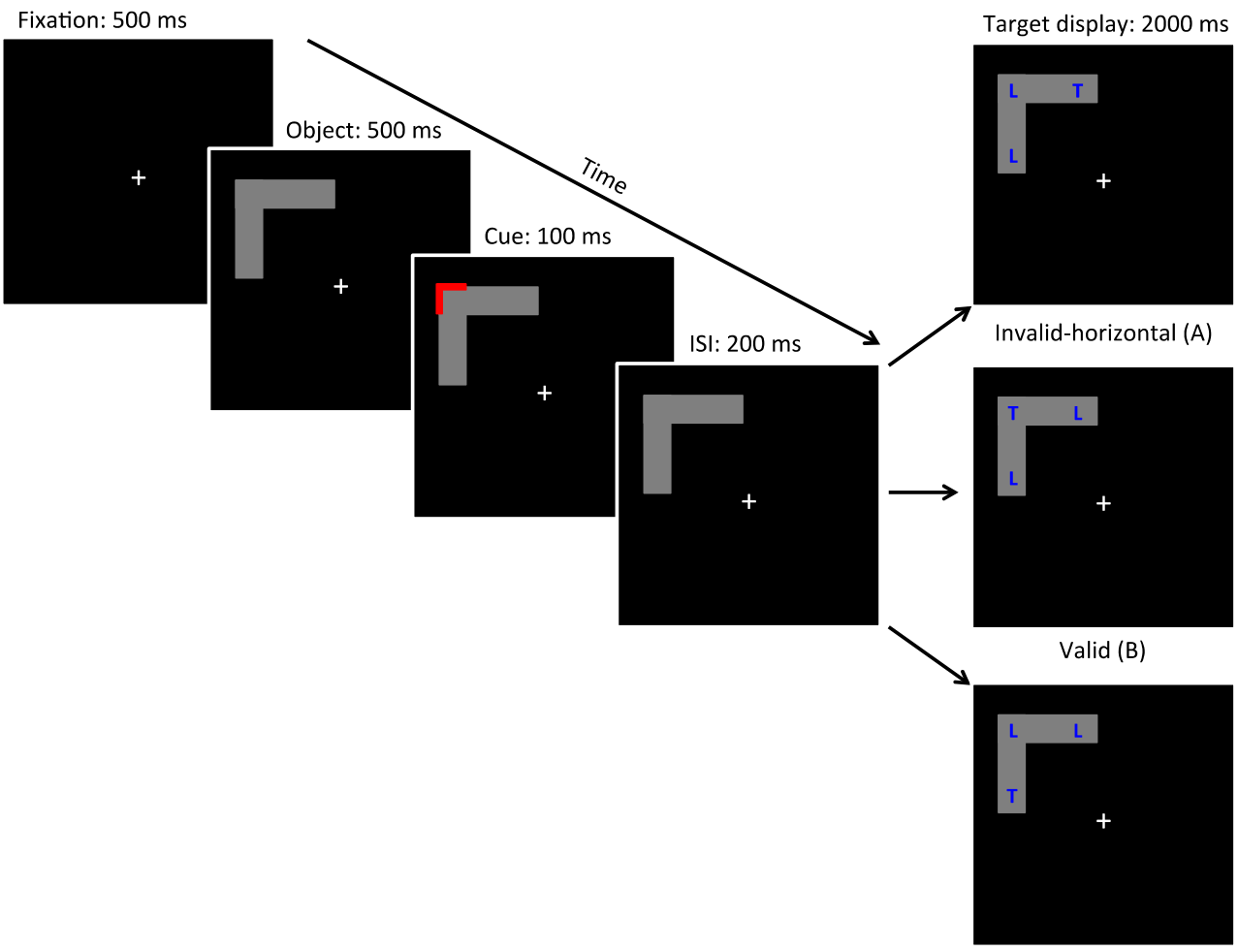

b

Invalid-vertical (C)

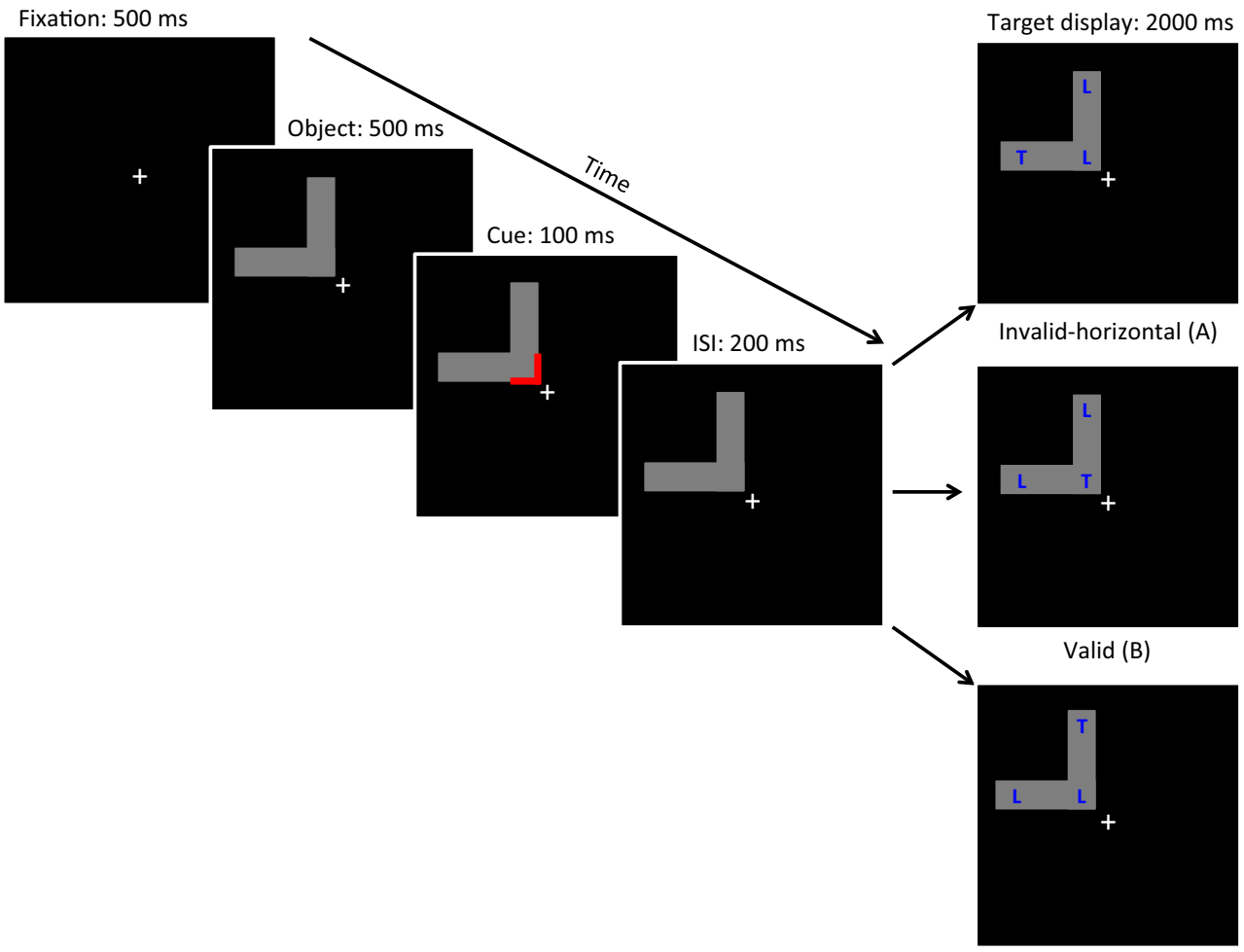

Invalid-vertical (C) 
horizontal trials (16 trials per block; 96 total), and 10\% invalid-vertical trials (16 trials per block; 96 total). The remaining condition was composed of "catch trials" $(20 \% ; 32$ trials per block; 192 total). These proportions were split evenly, such that both object types (those near fixation and those far from fixation) were allotted an equivalent number of trials (e.g., 288 valid trials for each object type).

\section{Results and discussion}

As in previous experiments, participants with false alarms to more than 19 catch trials were removed from the original sample of 41 participants before conducting any statistical analyses. This resulted in a final sample of 31 participants $\left(M_{\text {age }}=23.23\right.$ years, $S D_{\text {age }}=7.37$ years; 23 women, 8 men $)$ with a mean false-alarm rate of 12 catch trials $(S D=3)$. Anticipatory responses $(<200 \mathrm{~ms}$ ) and misses (not responding to the presence of a target) were excluded from the individual subject data. For near-cued objects, participants, on average, failed to respond to $3.19 \%$ of valid trials $(S E M=0.49 \%)$, $5.64 \%$ of invalid-horizontal trials $(S E M=0.61 \%)$, and $4.55 \%$ of invalid-vertical trials $(S E M=0.71 \%)$. For far-cued objects, participants, on average, failed to respond to $5.19 \%$ of valid trials $(S E M=0.98 \%), 5.26 \%$ of invalid-horizontal trials $(S E M=0.96 \%)$, and $5.73 \%$ of invalid-vertical trials $(S E M=$ $1.07 \%)$.

Mean RT differences were calculated by subtracting mean raw RTs to valid targets $(M=502.79 \mathrm{~ms}$ for near-cued objects; $M=598.93 \mathrm{~ms}$ for far-cued objects) from mean RTs to targets in each invalid location, and submitted to a $2 \times 2$ repeated measures ANOVA with Object Vertex Location (near fixation, far from fixation) and Shift Direction (vertical, horizontal) as within-subjects factors. The analysis revealed a main effect of Object Vertex Location, $F(1,30)=14.70, p=0.001, \eta_{\mathrm{p}}{ }^{2}=$ 0.33 , indicating a significant difference in target detection when reallocating object-based attention to invalid targets on objects that were cued near fixation $(M=266.49)$ versus objects that were cued far from fixation $(M=173.60 \mathrm{~ms})$. Furthermore, the analysis revealed a main effect of Shift Direction, $F(1,30)=20.99, p \leq 0.001, \eta_{\mathrm{p}}{ }^{2}=0.41$, indicating a significant difference in invalid target detection when reallocating attention horizontally $(M=199.64 \mathrm{~ms})$ versus vertically $(M=240.45 \mathrm{~ms})$. These main effects were further qualified by a significant two-way interaction, $F(1,30)=4.77$, $p=0.037, \eta_{\mathrm{p}}{ }^{2}=0.14$. Similar main effects of Object Vertex Location and Shift Direction ( $p s \leq 0.001)$, as well as the interaction $(p=0.001)$ were found when all 41 participants were analyzed together.

The interaction between Object Vertex Location and Shift Direction describes a significant difference in the shift direction magnitude as a function of object location (Fig. 5). Paired samples $t$ tests revealed that horizontal shifts $(M=163.82 \mathrm{~ms})$

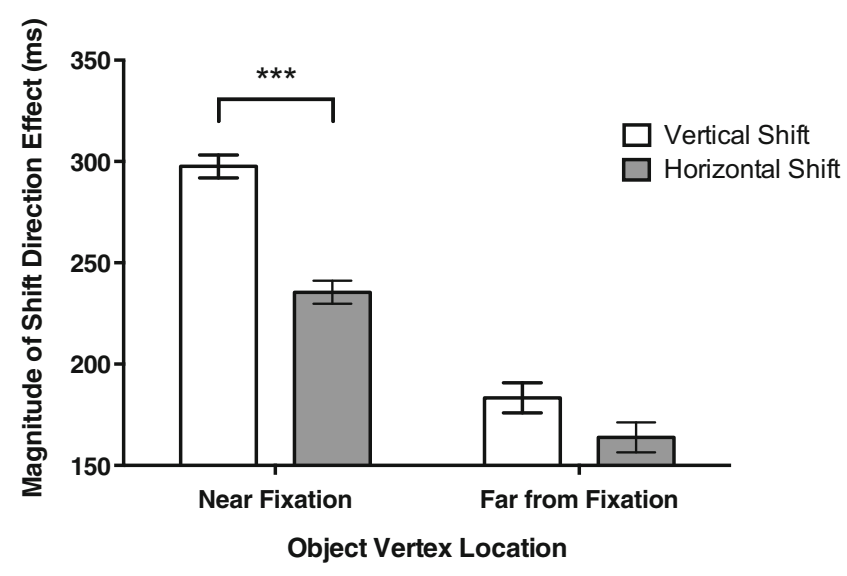

Fig. 5 Mean response time data ("Magnitude of Shift Direction Effect") measured for the Object Vertex Location x Shift Direction interaction in Experiment 3

were statistically equivalent to vertical shifts $(M=183.38 \mathrm{~ms})$ on objects cued far from fixation, $t(30)=1.32, p=0.197$, although we did observe a significantly larger shift direction effect for horizontal shifts $(M=235.46 \mathrm{~ms})$ compared with vertical shifts $(M=297.52 \mathrm{~ms})$ on objects cued near fixation, $t(30)=5.47, p \leq 0.001$. Thus, as shown in Table 1, the interaction is driven by a larger anisotropy between horizontal and vertical shifts for the near shift distance $(62.06 \mathrm{~ms})$ versus the far shift distance (19.56 ms).

Unlike Experiments 1 and 2, Experiment 3 revealed that shifts of object-based attention contained within the boundaries of a single cued L-shaped object that did not cross the screen meridians were allocated with equal efficiency along either the horizontal meridian or the vertical meridian. However, this effect only occurred when the object vertex (and spatial cue) was far from fixation; horizontal shifts were faster than vertical shifts when the object vertex was near fixation. These results suggest that there exist conditions under which inter- and intrahemispheric boundaries do not affect horizontal and vertical shifts of object-based attention in the absence of meridian crossings.

\section{General discussion}

Previous research has shown that the direction of OBA effects can be modulated by object orientation (Pilz, Roggeveen, Creighton, Bennett, \& Sekuler, 2012; but see Greenberg et al., 2014 and Al-Janabi \& Greenberg, under review for the current special issue). Those studies, specifically, showed evidence of a same-object advantage (i.e., faster RTs to the invalid location on a cued object versus a non-cued object) for horizontally oriented rectangles but not for vertically oriented rectangles. In fact, under certain conditions, a same-object cost (i.e., slower RTs to the invalid location on a cued object vs. a non-cued object) has been observed (Pilz et al., 2012). 
Following the assertion by Pilz et al. (2012) that horizontal shifts of object-based attention are more efficient, we hypothesized that this dissociation between object-based effects emerging from horizontal objects and not vertical objects could be due to more efficient allocation of attention along the horizontal meridian versus the vertical meridian. The mechanism we proposed as an explanation for this effect is the intrahemispheric boundary within retinotopic visual cortex that stems from the known dissociation between upper and lower visual field representations. Of course, the interhemispheric boundary (caused by the contralateral visual field organization of cortex) may also cause the visual field meridians to play a role in attention shift efficiency. Thus, the purpose of this paper was to examine, behaviorally, the manner in which shifts of object-based attention vary as a function of crossing the horizontal and vertical meridians.

We found, in Experiments 1 and 2, that the allocation of object-based attention within an object and between objects was more efficient along the horizontal meridian rather than the vertical meridian. In these experiments, the displayed objects were positioned such that they crossed both the horizontal and vertical visual field meridians. Participants detected the target faster when its location necessitated a horizontal shift of attention from a given cued location compared with a vertical shift of attention from a given cued location. This pattern of performance was observed when participants were required to shift attention across visual field meridians either within the cued object (Experiments 1 and 2) or between cued and noncued objects (Experiment 2). Importantly, our finding that OBA is more efficient for horizontal, rather than vertical, shifts of object-based attention mirrors results reported in studies of spatial attention (Carrasco, Evert, Chang, \& Katz, 1995; MacKeben, 1999), which show better performance along the horizontal meridian than along the vertical meridian.

In Experiment 3, we sequestered the object within one screen quadrant such that it did not cross either meridian. When the object vertex (and peripheral cue) was located far from central fixation, we no longer observed any difference in performance between horizontal and vertical shifts of attention. We attribute this finding to the strong influence that the horizontal and vertical meridians of the visual field exert on attentional selection. Because each of these meridians form a neurophysiological boundary (either inter- or intrahemispheric), shifts of attention that cross one of these meridians may require additional cortical processing in the form of hemispheric interactions or long-distance lateral connections. In particular, the anisotropy that we observed in Experiments 1 and 2 suggest that crossing the horizontal meridian, specifically, impairs the efficiency with which objectbased shifts of attention can occur. We, thus, provisionally conclude that the horizontal visual field meridian negatively impacts object-based attention performance when shifting between the upper and lower visual hemifields. By extension, when attention shifts do not require any meridian boundary crossings, no advantage emerges for horizontal shifts of object-based attention.

Importantly, we did find one condition in which neither visual field meridian was crossed and yet we still observed a horizontal-vertical anisotropy in object-based attention shift performance. When the object vertex (and peripheral cue) appeared in close proximity to central fixation, participants detected the target faster when it appeared horizontally rather than vertically, even though a meridian crossing was not required (Experiment 3). Thus, for objects that do not cross the meridians, when cued at a location in the periphery, no attentional shift anisotropy is observed, but when cued at a location near fixation, the anisotropy reemerges. We note that when cued near fixation, the object was oriented such that attending invalid target locations required shifts from (near) center to periphery adjacent to either the horizontal or vertical meridian. However, when cued far from fixation, the object was oriented such that attention was shifted between peripheral locations, moving toward either the horizontal or vertical meridian. These data are consistent with results from Reuter-Lorenz and Fendrich (1992b) in which they found that the performance decrement from invalid precues (as used herein) depends on cue location. Specifically, central cues evoked anisotropic effects due to visual field meridians, but peripheral cues did not. Therefore, we conclude that the horizontalvertical anisotropy we observed when the object was cued near central fixation was the result of using a (near) central cue and does not invalidate our previous conclusion that shifting object-based attention across the horizontal meridian is the primary cause of the performance decrement we observed in Experiments 1 and 2.

Nevertheless, it is clear that reallocating OBA across the visual field meridians, as well as in close proximity to them, will result in a horizontal shift advantage. This suggests a more general modulatory role of the meridians in affecting shift direction efficiency that is based on both meridian vicinity and crossings. We, thus, conclude that visual field meridians affect the efficiency with which OBA is allocated.

Our results also necessitate updating current theories of object-based attentional selection to account for crossings of the horizontal and vertical visual field meridians. Consider, for example, the attentional prioritization strategy proposed by Shomstein and Yantis (2002). They theorized that OBA is guided by an attentional prioritization strategy, whereby visual information contained within an attended (cued) object is afforded higher priority than visual information contained in an unattended (non-cued) object. Specifically, in the case of the double-rectangle cueing paradigm (Egly, Driver, \& Rafal, 1994), the prioritization strategy posits that objects are unequally prioritized for attentional resources, such that a higher prioritization is granted to the invalid location of the cued object, whereas a lower prioritization is granted to the invalid 
location of the non-cued object. This unequal setting of prioritization occurs even though both locations are equidistant from the cue, and suggests selection of the cued object over the non-cued object. It is reasonable to assume, based on this account, that OBA would be prioritized equally to both invalid locations (horizontal and vertical) in our L-shaped object, precisely because we are only ever comparing locations appearing on the same object. Interestingly, and contrary to this prediction, we found conditions under which prioritization of attention was unequally distributed across these locations. Indeed, our results indicate that in addition to prioritizing cued objects versus non-cued objects, observers may also prioritize dimensions of an object that appear horizontally rather than vertically, particularly when objects cross, or are in close proximity to, the visual field meridians.

\section{Limitations and future directions}

Across all three experiments, a consistent 300-ms cue-target SOA was used. It is possible that an SOA of that length allowed participants the opportunity to break fixation and make overt eye movements toward the cued location upon the onset of the peripheral cue. Although this does not invalidate our results, the issue of overt object-based selection requires further study (McCarley, Kramer, \& Peterson, 2002 and Şentürk, Greenberg, \& Liu, under review for the current special issue). It is important to note that the SOA utilized herein is consistent with that of many other published reports on OBA (Egly, Driver, \& Rafal, 1994; Moore, Yantis, \& Vaughan, 1998; Pratt \& Sekuler, 2001; Marino \& Scholl, 2005; Pilz et al., 2012; Greenberg et al., 2015), making it unlikely that our subjects were doing something significantly different from most other studies of object-based attention.

Additionally, participants were given $2000 \mathrm{~ms}$ to respond to the presence of the target "T." It also is possible that the length of the response window allowed participants the opportunity to make multiple saccades to the invalid-horizontal and the invalid-vertical target locations. If this is true, one might question whether the consistent horizontal shift advantage could be explained by faster horizontal eye movements compared with vertical eye movements, thus resulting in our observed shift direction anisotropies. First, the fact that we failed to observe the anisotropy in one condition makes it unlikely that eye movement directions could account for this effect since eye movements would have been equally likely in all conditions tested. Second, Becker and Jürgens (1990) found that horizontal and vertical saccades to targets in the cardinal directions (left, right, up, and down) had statistically equivalent peak velocities. Nevertheless, in consideration of the three experiments reported in this manuscript, the possibility that the shift direction anisotropy could be explained by faster horizontal eye movements compared with vertical eye movements would be substantiated by investigations of how saccadic reaction time is affected by the objects used in objectbased attention paradigms. As such, research conducted by Şentürk, Greenberg, and Liu (under review for the current special issue) examined differences between manual button press response times and saccadic eye-movement latencies to the presence of a target during the double-rectangle cueing paradigm (Egly. Driver, \& Rafal, 1994). In general, saccades to validly cued targets were faster than saccades to invalidly cued targets, and saccades to invalid-same object targets were significantly faster than saccades to invalid-different object targets (Șentürk et al.). These results of eye movements made in the context of object-based attention produced qualitatively similar effects to those produced by button presses, suggesting that both processes (manual responses and saccade latency) are affected similarly under conditions of object-based attentional selection.

Finally, in Experiment 3, our observed effect of object vertex location (near fixation and far from fixation) is conflated with target eccentricity. It was important to us that the cue be equidistant from the two screen meridians because of the purported role of visual field meridians in the shift direction anisotropy. Given that participants were instructed to fixate centrally, we were unable to differentiate between (a) the effect of object distance from the screen meridians, and (b) an effect of eccentricity. That is, near cues cannot be far from the screen meridians while also being equidistant from them; and far cues cannot be close to the screen meridians while also being equidistant from them. Future research may consider these effects during studies in which one is less bound by having participants fixate centrally and/or forcing cues to be equidistant from the two screen meridians.

\section{Conclusion}

This study sought to examine how the reallocation of objectbased attention within a cued object, and between cued and non-cued objects, varies as a function of crossing the horizontal and vertical meridians. Our results demonstrate that OBA is allocated unevenly within objects that cross the screen meridians, regardless of whether those shifts take place within a cued object or between two objects. These findings necessitate the updating of OBA theories to include effects of crossing the horizontal and vertical visual field meridians, as well as the locations of the object and cue within the visual field.

Acknowledgments The authors thank Richard Dubbelde III, Grace Nicora, and Nicole Kashian for their assistance with data collection and also Shahd Al-Janabi for her insightful comments. This research was supported by the United States-Israel Binational Science Foundation Grant No. 2013400 (ASG). 


\section{References}

Al-Janabi, S. \& Greenberg, A. S. (under review for the current special issue). Target-object integration, attention distribution, and object orientation interactively modulate object-based selection. Attention, Perception, and Psychophysics.

Bateman, D., Eaton, J. W., Wehbring, R., \& Hauberg, S. (2015). The GNU Octave 4.0 Reference Manual 1/2: Free Your Numbers. London: Samurai Media Limited.

Becker, W., \& Jürgens, R. (1990). Human oblique saccades: Quantitative analysis of the relation between horizontal and vertical components. Vision Research, 30(6), 893-920.

Brainard, D. H. (1997). The Psychophysics Toolbox. Spatial Vision, 10, 433-436. doi:10.1163/156856897X00357

Carrasco, M., Evert, D. L., Chang, I., \& Katz, S. M. (1995). The eccentricity effect: Target eccentricity affect performance on conjunction searches. Perception \& Psychophysics, 57(8), 1241-1261.

Chen, Z. (2012). Object-based attention: A tutorial review. Attention, Perception, \& Psychophysics, 74, 784-802. doi:10.3758/s13414012-0322-Z

Chen, Z., \& Cave, K. R. (2006). Reinstating object-based attention under positional certainty: The importance of subject parsing. Perception \& Psychophysics, 68, 992-1003. doi:10.3758/BF03193360

Chen, Z., \& Cave, K. R. (2008). Object-based attention with endogenous cuing and positional certainty. Perception \& Psychophysics, 70, 1435-1443. doi:10.3758/PP.70.8.1435

Chen, H., \& Huang, L. (2015). Solving the paradox between same-object advantage and different-object advantage. Vision Research, 115, 128-134. doi:10.1016/j.visres.2015.08.008

Davis, G., \& Holmes, A. (2005). Reversal of object-based benefits in visual attention. Visual Cognition, 12, 817-846. doi:10.1080/ 13506280444000247

Desimone, R., \& Duncan, J. (1995). Neural mechanisms of selective visual attention. Annual Review of Neuroscience, 18, 193-222. doi:10.1146/annurev.ne.18.030195.001205

Duncan, J. (1984). Selective attention and the organization of visual information. Journal of Experimental Psychology: General, 113, 501517. doi:10.1037/0096-3445.113.4.501

Egly, R., Driver, J., \& Rafal, R. D. (1994). Shifting visual attention between objects and locations: Evidence from normal and parietal lesion subjects. Journal of Experimental Psychology: General, 123, 161-177. doi:10.1037/0096-3445.123.2.161

Greenberg, A. S., Hayes, D., Roggeveen, A., Creighton, S., Bennett, P., Sekuler, A., \& Pilz, K. (2014). Object-based attention is modulated by shifts across the meridians. Journal of Vision, 14, 1062. doi:10. $1167 / 14.10 .1062$

Greenberg, A. S., Rosen, M., Cutrone, E., \& Behrmann, M. (2015). The effects of visual search efficiency on object-based attention. Attention, Perception, \& Psychophysics, 77, 1544-1557. doi:10. 3758/s13414-015-0892-7

Harrison, S. J., \& Feldman, J. (2009). Perceptual comparison of features within and between objects: A new look. Vision Research, 49, 2790 2799. doi:10.1016/j.visres.2009.08.014

Holtzman, J. D., Sidtis, J. J., Volpe, B. T., Wilson, D. H., \& Gazzaniga, M. S. (1981). Dissociation of spatial information for stimulus localization and the control of attention. Brain, 104(4), 861-872.
MacKeben, M. (1999). Sustained focal attention and peripheral letter recognition. Spatial Vision, 12(1), 51-72.

Marino, A. C., \& Scholl, B. J. (2005). The role of closure in defining the "objects" of object-based attention. Perception \& Psychophysics, 67, 1140-1149. doi:10.3758/BF03193547

McCarley, J. S., Kramer, A. F., \& Peterson, M. S. (2002). Overt and covert object-based attention. Psychonomic Bulletin \& Review, 9, 751-758. doi:10.3758/BF03196331

Moore, C. M., Yantis, S., \& Vaughan, B. (1998). Object-based visual selection: Evidence from perceptual completion. Psychological Science, 9(2), 104-110.

Pelli, D. G. (1997). The VideoToolbox software for visual psychophysics: Transforming numbers into movies. Spatial Vision, 10, 437-442. doi:10.1163/156856897X00366

Pilz, K. S., Roggeveen, A. B., Creighton, S. E., Bennett, P. J., \& Sekuler, A. B. (2012). How prevalent is object-based attention? PLoS One, 7(2), e30693. doi:10.1371/journal.pone.0030693

Posner, M. I. (1980). Orienting of attention. The Quarterly Journal of Experimental Psychology, 23(1), 3-25.

Posner, M. I., Snyder, C. R., \& Davidson, B. J. (1980). Attention and the detection of signals. Journal of Experimental Psychology, 109(2), $160-174$.

Pratt, J., \& Sekuler, A. B. (2001). The effects of occlusion and past experience on the allocation of object-based attention. Psychonomic Bulletin \& Review, 8(4), 721-727.

Reuter-Lorenz, P. A., \& Fendrich, R. (1992a). Orienting attention across the vertical meridian: Evidence from callosotomy patients. Journal of Cognitive Neuroscience, 2(3), 232-238.

Reuter-Lorenz, P. A., \& Fendrich, R. (1992b). Oculomotor readiness and covert orienting: Differences between central and peripheral precues. Perception \& Psychophysics, 52(3), 336-344.

Richard, A. M., Lee, H., \& Vecera, S. P. (2008). Attentional spreading in object-based attention. Journal of Experimental Psychology: Human Perception and Performance, 34, 842-853. doi:10.1037/ 0096-1523.34.4.842

Şentürk, G., Greenberg, A. S., \& Liu. T. (under review for the current special issue). Saccade latency indexes exogenous and endogenous object-based attention. Attention, Perception, and Psychophysics.

Sereno, A. B., \& Kosslyn, S. M. (1991). Discrimination within and between hemifields: A new constraint on theories of attention. Neuropsychologia, 29(7), 659-675.

Shomstein, S. (2012). Object-based attention: Strategy versus automaticity. WIREs Cognitive Science, 3, 163-169. doi:10.1002/wcs.1162

Shomstein, S., \& Yantis, S. (2002). Object-based attention: Sensory modulation or priority setting? Perception \& Psychophysics, 64(1), 4151.

Shomstein, S., \& Yantis, S. (2004). Configural and contextual prioritization in object-based attention. Psychonomic Bulletin \& Review, 111, 247-253. doi:10.3758/BF03196566

Van Essen, D. C. (1985). Functional organization of primate visual cortex. In A. Peters \& E. G. Jones (Eds.), Cerebral Cortex (Vol. 3, pp. 259-329). New York: Plenum.

Wolfe, J. M. (1994). Guided search 2.0: A revised model of visual search. Psychonomic Bulletin \& Review, 1, 202-238. doi:10.3758/ BF03200774 